\title{
Correction: Different enteral nutrition formulas have no effect on glucose homeostasis but on diet-induced thermogenesis in critically ill medical patients: a randomized controlled trial
}

\author{
Marlene Wewalka ${ }^{1} \cdot$ Andreas Drolz $^{1} \cdot$ Berit Seeland $^{1} \cdot$ Mathias Schneeweiss $^{1} \cdot$ Monika Schmid $^{1}$. \\ Bruno Schneeweiss ${ }^{2} \cdot$ Christian Zauner $^{1}$
}

Published online: 6 August 2018

(c) Springer Nature Limited 2018

Correction to: European Journal of Clinical Nutrition https://doi.org/10.1038/s41430-018-0111-4; published online 19 February 2018

After publication, the author noticed that Table 2 was incorrectly formatted for the final PDF despite being correct in earlier proofs. The table was correct in the HTML version of the article. The EJCN apologizes for the inadvertent error in the formatting of Table 2. The corrected version is uploaded and should be read in conjunction with the original paper. Any inconvenience to the author and readership is regretted.
Marlene Wewalka

marlene.wewalka@meduniwien.ac.at

1 Medical University of Vienna, Department of Internal Medicine III, Division of Gastroenterology and Hepatology, Intensive Care Unit, Waehringer Guertel 18-20, 1090 Vienna, Austria

2 Department of Internal Medicine, LKH Kirchdorf, Hausmanningerstrasse 8, 4560 Kirchdorf, Austria 